\title{
TOUCHINGbase
}

\section{Nature Genetics}

Barbara Cohen, the former editor of Nature Genetics, has accepted a position with a charitable endowment fund, the Ludwig Cancer Research Foundation, where she will head the Office of Communications. Bette Phimister, a veteran of 3.5 years with the journal, has been appointed editor and, together with Michael Ronemus (Associate Editor), Lawrence Jaskaran (Senior Editorial Assistant), Cara Hunsberger (Copy Editor) and Ingrid McNamara (Production Editor), looks forward to welcoming Tanguy Chouard to the Nature Genetics team. Tanguy comes from the laboratory of S. Lawrence Zipursky (University of California, San Diego), where he has been busy identifying components of neurological pathways in Drosophila.

\section{Reading the beads}

What do you get when you cross serial analysis of gene expression (SAGE), DNA-coated microbeads and a fluorescence-activated cell sorter? The answer: massively parallel signature-sequencing on microbead arrays. As reported in the pages of this month's issue of Nature Biotechnology (vol. 18, 630-634; 2000) Sydney Brenner (Lynx Therapeutics) and colleagues have generated a 'microarray' with a million probes of known sequence to analyse mRNA. A 'population' of beads, each with approximately 100,000 copies of a specific SAGE tag attached to the surface of each bead, is introduced into a narrow flow cell, creating a monolayer in which each bead occupies a unique position. Through an iterative application of techniques including linker ligation, enzymatic cleavage and interrogation with specific, fluor-labelled adapters, they are able to determine the identities of four or five specific 4-nucleotide sequences, and thus the 16-20 nucleotide 'signature' of the DNA on each bead. The position and signature of each bead is then imaged digitally, and the number of beads with each signature quantified. The hope is that such a large population of sequences will enable the detection of rare messages with relative ease.

\section{Spruce Goose 2000}

The Howard Hughes Medical Institute (HHMI) has announced the appointment of 48 new investigators, who will join the current group of $305 \mathrm{HHMI}$ investigators working at a number of institutions throughout the United States.

The new appointees were selected from 430 nominees following the recommendations of a review committee; each will receive approximately 1 million dollars (US) per year for research, support and training costs. Of particular note in this competition was the selec-

tion-for the first time-of 12 investigators in the up-and-coming field of computational biology. Among the 48 new investigators are David Haussler (University of California, Santa Cruz), Bruce T. Lahn and Daphne Preuss (University of Chicago), Jeannie T. Lee (Harvard Medical School), Craig C. Mello (University of Massachusetts Medical School), Sean R. Eddy (Washington University), Matthew L. Warman (Case Western Reserve University), Nancy M. Bonini (University of Pennsylvania), Bradley R. Cairns (University of Utah), Philip Green (University of Washington), and Leonid Kruglyak and Cecilia B. Moens (Fred Hutchinson Cancer Research Center). Congratulations to them and all of the other appointees.

There's been a bit of pop-psychology talk lately about thrill-seeking behavior like big-wave surfing being some sort of genetic thing... the way criminal behavior and depression and alcoholism are genetic. If it weren't for Mavericks and Waimea and other such places, some of these guys would be dead or in jail or in rehab. Society should be glad for big waves.

-Stone Parker, Surfer Magazine

\section{Gone fishing}

The ranks of the zebrafish community are swelling, as indicated by the attendance of around 500 participants at April's meeting at the Cold Spring Harbor Laboratory. The zebrafish mutant community may be swelled by a new venture: the Tübingen 2000 Screen project. Investing approximately \$10 million, Artemis Pharmaceuticals, together with the Max-Planck Society (which is a shareholder and co-owner of Artemis), has launched a mutagenesis programme through which they expect to generate and screen about 17 million mutant fish. Researchers from other centres are also participating in the project: at the end of the fishfest in approximately a year's time, the mutants will be divided up, with each party taking home mutants that suit its own interests. Whereas the previous zebrafish screen carried out in Tübingen focused on the identification of fish with defects in early patterning events, the new project will place emphasis on the identification of those with phenotypes that manifest later in life-for example, those involving aberrations in behaviour. Artemis will retain exclusive commercial rights to all discoveries made through material transfer agreements, an arrangement that has been applied retroactively, to the dismay of some, to mutants generated through previous screens.

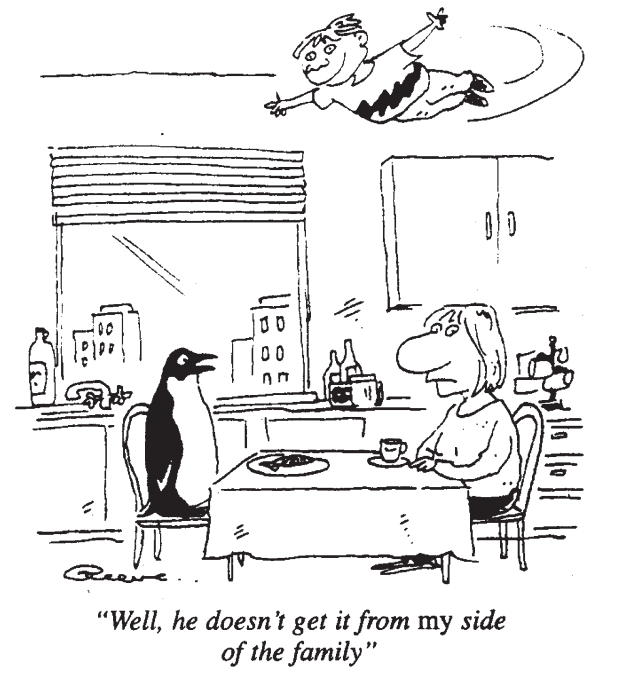

